\title{
Gear fault diagnosis method of intelligence based on genetic algorithm to optimize the BP neural network
}

\author{
Jie Fang \\ Department of Maths and Computer Science \\ Fuqing Branch of Fujian Normal University \\ Fuqing, China \\ E-mail: fangjie_1@163.com
}

\begin{abstract}
The work of the gear transmission is very complex, and its failure in the form and features tend to show non-linear mapping. Fault signal is often submerged in conventional vibration signal and noise, it is not easy using traditional signal processing methods to extract fault features which in a difficult to gear fault diagnosis. This paper based on the genetic algorithm to optimize the structure of the BP neural network model for the intelligent diagnosis system which is used in gear fault diagnosis.The experimental results show that this method can be effectively used for the diagnosis and identification of the gears common fault type.
\end{abstract}

Keywords-Artificial Neural; BP neural network; Gear fault intelligent diagnosis; Vibration Signal

\section{INTRODUCTION}

The gearbox is widely used in various types of running machinery which is the most commonly component used for variable speed and transfer power. Gear as one of the important parts of the gearbox, especially the operating status directly affect the performance of the whole machine, so gear fault diagnosis technology has important practical value and practical significance. Neural Network with its distributed parallel information processing, adaptive self-learning and associative memory, etc, to solve the fault diagnosis system of knowledge, access and parallel reasoning opened up new ways, one of the most widely applied is BP neural network. There are some defects of BP neural network, such as learning convergence is slow, can not guarantee convergence to the global minimum, the network structure is not easy to stability. In addition, the network structure, initial connection weights and thresholds of the choice have a great impact on the network training, but can not get accurately. Genetic algorithm can be used to optimize the neural network for these defects.

\section{BP NEURAL NETWORK THEORY}

\section{A. Artificial Neural}

The artificial neuron (or neurons) is a biological neuron simple imitation, simplification and abstraction, it is a very simple calculation unit (function). Figure 1 is the most typical MP (artificial neuron model) which is proposed by McCulloch (neuroanatomist) and Pitts (mathematician) in 1943.
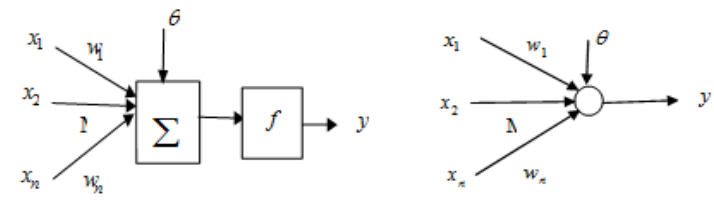

Figure 1. Artificial neural model

Neurons achieve a very simple non-linear function of $R^{n} \rightarrow R^{1}$ :

$$
y=f\left(\sum_{i=1}^{n} w_{i} x_{i}-\theta\right)=f(W \mathbf{x}-\theta)
$$

$$
f(x)= \begin{cases}1, & \text { if } x \geq 0 \\ 0, & \text { otherwise }\end{cases}
$$

Following show a logical function AND operator by using artificial neural. 1-True, 0-False.

TABLE I. THE INPUT AND OUTPUT OF THE NEURON BY LOGIC AND

\begin{tabular}{|c|c|c|}
\hline \multicolumn{2}{|c|}{ Neurons Input } & Neurons Output \\
\hline$X_{1}$ & $x_{2}$ & $y$ (AND) \\
\hline 0 & 0 & 0 \\
\hline 1 & 0 & 0 \\
\hline 0 & 1 & 0 \\
\hline 1 & 1 & 1 \\
\hline
\end{tabular}
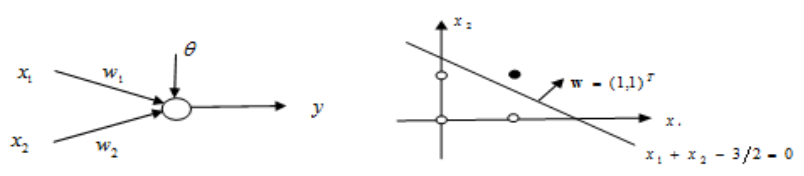

Figure 2. Artificial neuron achieve the logic gates

$$
\begin{aligned}
& \text { this } \\
& f\left(x_{1}+x_{2}-3 / 2\right)= \begin{cases}1, & \text { if } \mathbf{x}=(1,0)^{\mathrm{T}},(0,1)^{\mathrm{T}},(0,0)^{\mathrm{T}} \\
0, & \text { if } \mathbf{x}=(1,1)^{\mathrm{T}}\end{cases}
\end{aligned}
$$




\section{B. BP neural network}

BP neural network is a multilayer feedforward neural networks, the topology shown in Figure 1. The main features of the network signal to pass the error back propagation. To transfer in the former, the input signal from the input layer to hidden layer, layer by layer processing until the output layer. Neurons status of each layer affects only the state of the next layer of neurons. If the output layer are not expected output, then transferred to the back-propagation to adjust the network weights and thresholds of the prediction error, so that the BP neural network output closer and closer to the desired output. BP neural network topology shown in Figure 3.

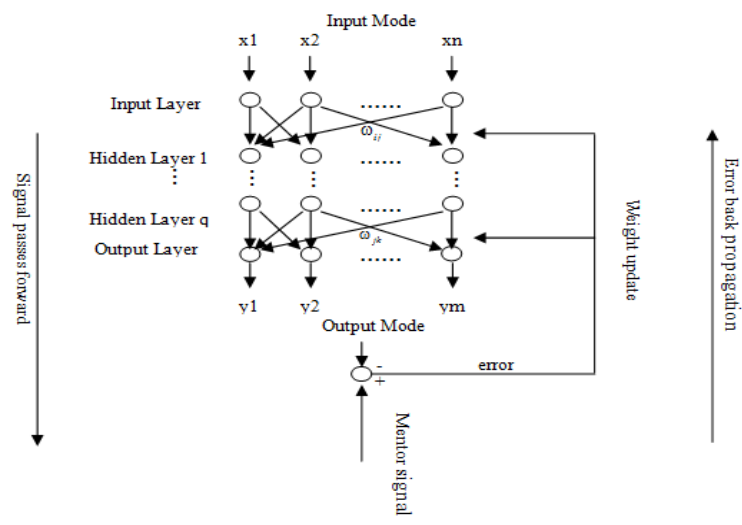

Figure 3. BP neural network topology

In Figure $3, X_{1}, X_{2}, \ldots, X_{n}$ is the input value of the BP neural network, $Y_{1}, Y_{2}, \ldots, Y_{m}$ is the BP neural network predictive value, $\omega_{i j}$ and $\omega_{j k}$ are value for the BP neural network weights.

\section{GENETIC ALGORITHMS}

The genetic algorithm (genetic algorithm) is a simulation of natural evolutionary processes search the optimal solution. It draws on the biological natural selection and natural genetic mechanisms by J. H. Holland Professor in the University of Michigan on 1960s. It is based on Darwin's survival of the fittest, the evolutionary principle about survival of the fittest, the basic operation of the repeated use of the genetics of the group that contains the feasible solution, and continuously generate a new group, the populations evolve.

\section{A. The basic mechanism of the genetic algorithm}

The genetic algorithm inspired by biological genetic evolution, the formation of a unique way for optimization, and its operation principle is usually consistent with the biological theory of genetic evolution, otherwise the term is often modeled the biological terminology. It adaptive process portrayed in changing the structure of space in the search process, that: (1) many of the complex structure can use the simple form of encoding through the bit string to represent; (2)it can be improved these bits string structure by some simple transformations, so change it to the desired direction, and then designed genetic algorithm to achieve the structure of the evolutionary process.

\section{B. Genetic algorithm implementation process}

In this case, we use Sheffield genetic algorithm toolbox for the genetic algorithm. The basic elements of the genetic algorithm include chromosome encoding method, fitness function, genetic manipulation and operation parameters.

(1) Treating solve the problem of encoding, the chromosome encoding.Assume that a parameter range from $\mathrm{A}$ to $\mathrm{B}, \mathrm{A}>\mathrm{B}$. Using binary encoding which the length is $l$ to indicate the parameters, make the elements in $[\mathrm{A}, \mathrm{B}]$ divided into $2^{l}-1$ sub-part, each identical length is $\delta$, it can produce $2^{l}$ different encoding, the corresponding relations of the parameters are as follows:

$$
\begin{aligned}
& 00000000 \cdots \cdot \cdots 0000000=0 \quad->\mathrm{A} \\
& 00000000 \cdots \cdots 0000001=1 \quad->\mathrm{A}+\delta \\
& \vdots \quad \vdots \quad \vdots \\
& 11111111 \cdots \cdots \cdot 11111111=2^{1}-1-->B
\end{aligned}
$$

(2) Random initialization groups, and determine the population size $M$, genetic algebra $G$, the crossover probability $P_{c}$ and mutation probability $P_{m}$.

(3) TSP problem, for example, the goal is find the shortest path in the total path length. Naturally, the total path length can become the fitness function for the TSP problem.

$$
f\left(w_{1} w_{2} \cdots w_{n}\right)=\frac{1}{\sum_{j=1}^{n} d\left(w_{j}, w_{j+1}\right)}
$$

(4) Pre-selection of operator to produce offspring, and offspring genetic manipulation. There are three main genetic manipulation: selection, crossover, mutation.

Select operation, according to the individual fitness function value measured by the degree of the quality to make a decision whether individuals in the next generation to be eliminated or genetic. General, the adaptive larger (excellent) individuals have a greater probability, the other has less probability. Simple crossover operator will be selected out of two individuals $P_{1}$ and $P_{2}$ as parents, exchange some code value in them.

Suppose the following two individuals, each of them have 8 bit.

\begin{tabular}{|l|l|l|l|l|l|l|l|l|}
\hline$P_{1}$ & 1 & 0 & 0 & 0 & 1 & 1 & 1 & 0 \\
\hline$P_{2}$ & 1 & 1 & 0 & 1 & 1 & 0 & 0 & 1 \\
\hline
\end{tabular}

Generate a random number $\mathrm{c}$ between 1 and 7 , if production is 3, and exchange the low three of $\mathrm{P} 1$ and $\mathrm{P} 2$ : the high five of the P1 and the low three of P2 composed the number string 10001001, which is an offspring $Q_{1}$ of 
the $P_{1}$ and $P_{2}$; the high five of $\mathrm{P} 2$ and the low three of $\mathrm{P} 1$ composed the number string 11011110, and another offspring $Q_{2}$ of the $P_{1}$ and $P_{2}$. Exchange process as shown in Figure 4.17.
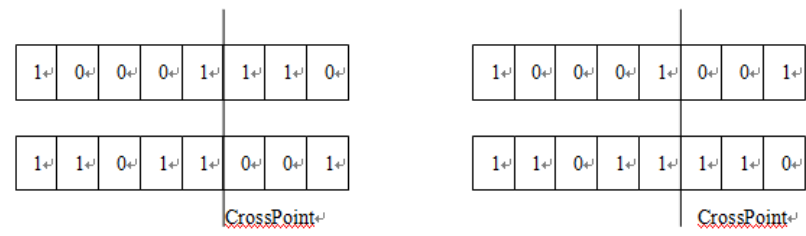

Figure 4. Cross-point of operation

The mutation operation, a simple way to digital in a position to change the digital string. The binary coded represent a simple mutation operation is that 0 and 1 are interchangeable. The following binary coded representation:

\begin{tabular}{|l|l|l|l|l|l|l|l|}
\hline 1 & 0 & 1 & 0 & 0 & 1 & 1 & 0 \\
\hline
\end{tabular}

Its code length is 8 , randomly generated a number $\mathrm{k}$ between 1 to 8 , if $k-=5$,make mutation operation for the fifth from right to left, let the original 0 to 1 , the following digital string (the fifth number is 1 after mutation operation):

\begin{tabular}{|l|l|l|l|l|l|l|l|}
\hline 1 & 0 & 1 & 1 & 0 & 1 & 1 & 0 \\
\hline
\end{tabular}

(5)Determine whether to meet the stop conditions, if not go to step 3, otherwise output the best adaptive individual in the groups.

\section{The complete code}

\section{GENETIC ALGORITHM TO OPTIMIZE THE BP NEURAL NETWORK APPLIED TO THE GEAR FAULT DIAGNOSIS}

A. Fault diagnosis model based on genetic algorithm to optimize the BP neural network

BP neural network topology based on the number of input / output parameters of the sample data, with the number of weights and thresholds will be determined, but they tend to have a significant impact on the network training, so it is necessary to use genetic algorithm to optimize the initial weights and thresholds. The genetic algorithm optimization BP neural network fault diagnosis model shown in Figure 5.

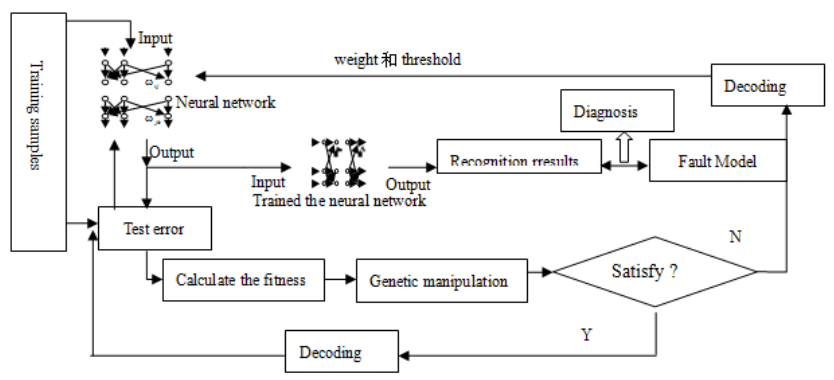

Figure 5. BP neural network fault diagnosis model

\section{B. Example}

In gear fault diagnosis experiment, we will have three specified categories of data acquisition, after the time-domain analysis, the amplitude domain analysis, the parameters of the extraction of amplitude domain and frequency domain parameters of neural network training samples, then construct $\mathrm{BP}$ neural network learning and diagnosis in MTALAB environment.

\section{(1) Sample data}

Due to the frequency domain, gear failure meshing frequency at the edge of the belt, so select in the frequency domain characteristics of signals extracted in the three stalls in the 1,2,3 axis side-band family at the amplitude, which is just a nine-dimensional vector. Select the six groups for normalized the Gears state after a treatment sample data as input to the neural network, as shown in Table 2.

TABLE II. INPUT SAMPLE DATA

\begin{tabular}{|c|c|c|}
\hline $\begin{array}{c}\text { Sample } \\
\text { number }\end{array}$ & Sample characteristic data & $\begin{array}{c}\text { The meaning of } \\
\text { characteristics }\end{array}$ \\
\hline 1 & 0.1150 .2270 .0610 .2580 .1140 .2390 .227 & crack \\
\hline 2 & 0.3110 .266 & trouble-free \\
\hline 3 & $0.1200 .070 \begin{array}{c}0.0110 .1230 .2660 .0770 .180 \\
0.1290 .123\end{array}$ & broken teeth \\
\hline 4 & 0.2220 .0560 .3220 .0330 .0820 .1910 .208 & crack \\
\hline 5 & 0.1340 .6570 .0610 .2620 .2580 .1150 .151 \\
0.2270 .322 & trouble-free \\
\hline 6 & 0.2310 .6520 .0810 .1630 .2230 .1210 .182 \\
0.1020 .137 & broken teeth \\
\hline
\end{tabular}

In addition, choose the other three groups for normalized the Gears state after a treatment sample data as test data, as shown in Table 3.

TABLE III. CHARACTERISTIC FACTOR OF THE INPUT LAYER NODE VALUES

\begin{tabular}{|c|c|c|}
\hline $\begin{array}{c}\text { Sample } \\
\text { number }\end{array}$ & Sample characteristic data & $\begin{array}{c}\text { The meaning } \\
\text { of } \\
\text { characteristics }\end{array}$ \\
\hline 1 & 0.0990 .2100 .0810 .0380 .1000 .0750 .151 & trouble-free \\
\hline 2 & 0.2330 .2590 .0620 .1290 .1510 .2850 .254 & crack \\
\hline 3 & 0.3200 .2230 .12590 .3200 .0620 .0070 .156 & broken teeth \\
\hline
\end{tabular}

There are three kinds of fault modes, as shown in Table 4.

TABLE IV. FAULT MODE

\begin{tabular}{|c|c|c|}
\hline node numbers & The meaning of characteristics & $\begin{array}{c}\text { characteristic } \\
\text { value }\end{array}$ \\
\hline 1 & trouble-free & True-1, False-0 \\
\hline 2 & broken teeth & True- 1, False-0 \\
\hline 3 & crack & True- 1 , False-0 \\
\hline
\end{tabular}

(2) Data set of the genetic algorithm

Individual coding using binary encoding, each individual represents a binary string. In this case, the network structure is 
9-19-3, the number of its weights and threshold values shown in Table 5.

TABLE V. THE NUMBER OF WEIGHT AND THRESHOLD

\begin{tabular}{|c|c|c|c|}
\hline $\begin{array}{c}\text { Input layer } \\
\text { and hidden } \\
\text { layer } \\
\text { connection } \\
\text { weight }\end{array}$ & $\begin{array}{c}\text { hidden } \\
\text { layer } \\
\text { weight }\end{array}$ & $\begin{array}{c}\text { Hidden layer and } \\
\text { output layer } \\
\text { connection } \\
\text { weight }\end{array}$ & $\begin{array}{c}\text { output } \\
\text { layer } \\
\text { weight }\end{array}$ \\
\hline 365 & 19 & 93 & 5 \\
\hline
\end{tabular}

In this case the running parameter settings of genetic algorithm shown in Table 6.

TABLE VI. RUNNING PARAMETER SETTINGS OF GENETIC ALGORITHM

\begin{tabular}{|c|c|c|c|c|c|}
\hline $\begin{array}{c}\text { Population } \\
\text { size }\end{array}$ & $\begin{array}{c}\text { Maximum } \\
\text { genetic } \\
\text { algebra }\end{array}$ & $\begin{array}{c}\text { Variable } \\
\text { binary } \\
\text { digits }\end{array}$ & $\begin{array}{c}\text { Crossover } \\
\text { probability }\end{array}$ & $\begin{array}{c}\text { Mutation } \\
\text { probability }\end{array}$ & gap \\
\hline 40 & 50 & 10 & 0.7 & 0.01 & 0.95 \\
\hline
\end{tabular}

(3) Create Network

As BP neural network for fault diagnosis is concerned, the input layer cell number generally equals to the dimension of characteristic vector, the output layer cell number equals to the number of fault category.

\section{(4) Network training and testing}

For example, the number of training times is 1000 , the training target is 0.01 , and the learning rate is 0.1 . After the optimization of genetic algorithm, we get a best initial weight and threshold matrix, the initial weights and thresholds of return and substituted into the BP neural network, Matlab experiments to draw the training error curve, predicted values, prediction error, training error and so on.

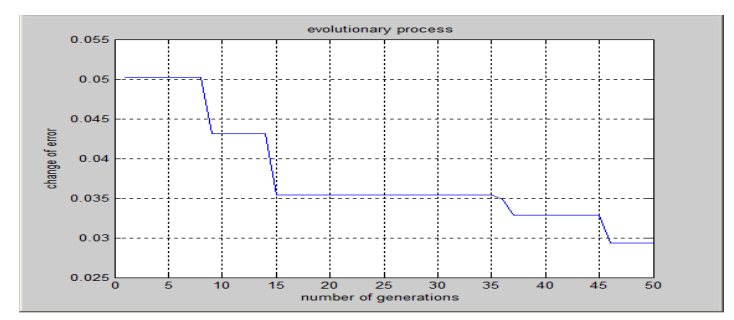

Figure 6. Error evolution curve

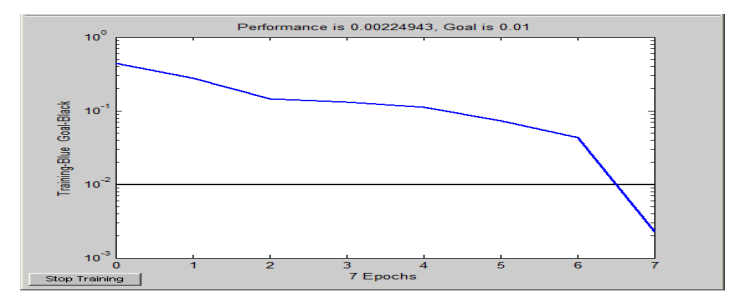

Figure 7. Random weights and thresholds of the training error curve

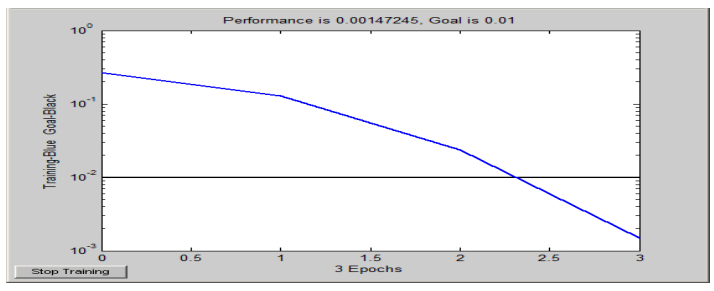

Figure 8. Optimized weights and thresholds of the training

It can be seen that the state of diagnosis is consistent with the actual fault condition, three kinds of test failures compound the actual situation. After the training of BP neural network is expected to output data and the error between the actual value, and has a high recognition accuracy can reach the gear fault diagnosis. One step closer to the initial weights and thresholds of BP neural network by genetic algorithm to optimize the error of the test sample and training sample error are greatly reduced, the training and prediction have been greatly improved.

\section{CONCLUSIONS}

Building BP neural network model based on adaptive genetic algorithm optimization, to study and training the $\mathrm{BP}$ neural network through the collection of gear fault feature parameters on gear fault diagnosis are effective. In addition to the genetic algorithm, we can use particle swarm optimization, ant colony algorithm or other algorithm to initialize weights and thresholds of BP neural network, this will be a research direction in the future.

\section{REFERENCES}

[1] Hu Shouren. Introduction to Neural Networks [M].Chang Sha: National Defense University Press, 1993 : 113-120.

[2] Jiang Liangxiao, Li Chaoqun. [J].MICROCOMPUTER \& ITS APPLICATIONS, 2004,(1): 52-53.

[3] Tian Jingwen, Gao Meijuan. Research and application of artificial neural network algorithm [M]. BEIJING INSTITUTE OF TECHNOLOGY PRESS, 2006.

[4] Li Zhihua,Wang Xiyang. Application of Wavelet Analysis and ANN on Cutter Fault Diagnosis[J]. MANUFACTURING TECHNOLOGY \& MACHINE TOOL,2005,(8):86 90

[5] Zhang Guobin, The Improvement and Application in Wavelet Neural Network Algorithm[D], Fuzhou University, 2005.

[6] Chen Zhe, Feng Tianjin,Chen Gang. A kind of BP Algorithm-Learning Wavelet Neural Network[J]. JOURNAL OF OCEAN UNIVERSITY OF QINGDAO:2001, 31(1) : 122 128.

[7] He Fei, Zhang Guozhong, Liu Ya. Improved load forecasting method based on BP network[J].EAST CHINA ELECTRIC POWER, 32(3) : 31-33.

[8] Chen Z, Feng T J, Meng Q C. The Application of Wavelet Neural Network in Time Series Prediction and System Modeling Based on Multiresolution Learning [J]. IEEE Conference on Systems, Man, and Cybernetics, 1999,(1): 425-430.

[9] Neural network theory and Mtalab 7 [M]. BEI JING: Publishing House of Electronics Industry, 2005. 